\title{
Determinación de los Factores del Peor Escenario en la Emisión de Gases Tóxicos
}

Christian O. Díaz-Ovalle ${ }^{1}$, Richart Vázquez-Román ${ }^{1 *}$ y M. Sam Mannan ${ }^{2}$

(1) Instituto Tecnológico de Celaya, Departamento de Ingeniería Química, Av. Tecnológico s/n, 38010 Celaya, Guanajuato-México (e-mail: richart@iqcelaya.itc.mx)

(2) Texas A\&M University, Artie McFerrin Department of Chemical Engineering, Mary Kay O'Connor Process Safety Center, College Station, Texas 77843-3122, U.S.A.

*autor a quien debe ser dirigida la correspondencia

\section{Resumen}

Se analizan modelos de dispersión convencionales para determinar los factores que incrementan el riesgo de muerte durante una emisión de gases tóxicos. El análisis se basa en la determinación de la concentración del gas tóxico en función de la distancia con respecto al punto de emisión. Así, el daño sobre una persona depende de esta concentración y del tiempo de contacto. Se muestra que el viento es un factor importante en la determinación del peor escenario. Además, las características del terreno modifican de manera importante la dispersión del gas. Los resultados indican que el peor escenario se tiene con viento en calma y con condición atmosférica estable en terreno rural. Los modelos de Pasquill-Gifford en dispersión pasiva y Germeles en dispersión de gas denso tienden a sobreestimar la concentración lo cual permite hacer estimaciones más seguras.

Palabras clave: dispersión de gases, factores de dispersión, modelos de dispersión, velocidad del viento, condición atmosférica

\section{Determination of the Factors for the Worst Scenario in Toxic Gas Release}

\begin{abstract}
Conventional dispersion models are analyzed to determine the factors that increase the risk of death during gas toxic release. The analysis is based in determining the toxic gas concentration as a function of the distance from the emission point. Thus, the injury in a person would depend on this concentration and the exposure time. It is shown here that the wind is an important factor to define the worst scenario. In addition, terrain characteristics substantially modify the gas dispersion. The results suggest that the worst scenario appears with calm wind and with stable atmospheric condition on rural terrain. The Pasquill-Gifford model in passive dispersion and Germeles model in gas dense dispersion tend to overestimate the concentration yielding safer estimations.
\end{abstract}

Keywords: gas dispersion, dispersion factors, dispersion models, wind speed, atmospheric condition 


\section{INTRODUCCION}

La estimación de la concentración de un gas en dispersión en el espacio proviene de la solución de la ecuación de difusividad convectiva (Crowl y Louvar, 2002). La solución analítica del modelo de dispersión es compleja y su aplicación se dificulta por la falta de valores correctos del coeficiente de difusividad respecto a las coordenadas espaciales y el tiempo. Por esta razón, varios modelos han sido desarrollados para evitar el uso del coeficiente de difusividad. En general, la dispersión se ha clasificado como pasiva y de gases densos (Mannan, 2005a; 2005b).

La dispersión pasiva se tiene cuando el gas en dispersión muestra comportamientos de flotación o inmersión en el aire. Una solución analítica a la ecuación de difusividad convectiva en estado transitorio fue desarrollada por Roberts en 1923, para analizar el comportamiento de gases de emisión en chimeneas (Mannan, 2005c). Esta propuesta fue modificada por Sutton en 1953, para aproximar el comportamiento de la dispersión a una forma gaussiana (Mannan, 2005c). En este modelo los parámetros de dispersión son obtenidos experimentalmente. Asimismo, Pasquill en 1961 propuso un modelo que estima la concentración considerando el valor de la desviación estándar de las concentraciones en dirección perpendicular a la dirección del viento (Mannan, 2005c). La desviación estándar en el modelo de Pasquill es obtenida de manera experimental y se ajustó a expresiones analíticas por Gifford en 1961 para generar así el modelo conocido como Pasquill-Gifford (Mannan, 2005c). Algunos experimentos realizados de forma pasiva determinaron valores aproximados para los parámetros de dispersión en caso de turbulencias como lo desarrollado por Gifford en 1976 (Mannan, 2005d), incluyendo el comportamiento de dispersión en zonas urbanas (Turner, 1964). Recientemente, los modelos modificados de Pasquill-Gifford se han usado para analizar la dispersión en zonas costeras (Park y Seok, 2007) y para calcular la distancia a la cual se tiene una concentración dada en un tiempo específico después de una emisión (Thomson et al., 2007).

Los gases densos no siguen, en un inicio, el patrón descrito en la dispersión pasiva debido al efecto de gravedad. Sin embargo, la nube en dispersión presenta un fenómeno de flotación tal que, después de una cierta distancia, se comporta como los gases menos pesados que el aire demostrado por van Ulden en 1974 (Mannan, 2005a). Los parámetros de dispersión propuestos por Gifford han sido adaptados a modelos de gases densos, en particular el modelo de Cox-Roe y Germeles (Mannan, 2005e). Algunos modelos son exclusivos de dispersiones instantáneas que incluyen un estado transitorio en la dispersión como el modelo DENZ (Mannan, 2005e). Un análisis particular de la influencia de la temperatura sobre la densidad donde la concentración se modela unidimensionalmente es propuesto en el modelo SLAB (Mannan, 2005e). Por otro lado, la solución simultánea de las ecuaciones de transferencia de momentum, calor y masa describiendo la dispersión de gases densos en tres dimensiones es aplicada en el modelo FEM3 (Ermak et al., 1982). Algunos modelos permiten obtener soluciones más precisas a través de una aplicación matemática más eficiente, e.g. HEGADAS y DEGADIS (Mannan, 2005e). Los experimentos realizados por el Lawrence Livermore Nacional Laboratory en el lago China, California, permitieron comparar los valores obtenidos en el modelado de dispersión de gases densos con los modelos Germeles, SLAB y FEM3 (Ermak et al., 1982). Estos experimentos concluyen que los modelos aseguran resultados cercanos a los reales. Actualmente los modelos de dispersión de gases densos son utilizados para mostrar el comportamiento de nubes de gases tóxicos (Dandrieux-Bony et al., 2005; Jonsson et al., 2005; Sklavounos y Rigas, 2004; Yegnan et al., 2002).

La ubicación de la concentración de un gas tóxico en dispersión permite llevar a cabo acciones preventivas y de diseño industrial (Vázquez-Román et al., 2007). Los accidentes industriales sobre liberaciones de gases tóxicos muestran la necesidad de la predicción de los accidentes para disminuir el grado de afectación, como en Bhopal, India (Sharan y Gopalakrishnan, 1997). El viento es una variable importante cuya intensidad es en realidad un valor altamente estocástico por lo que el cálculo del riesgo de afectación se vuelve una evaluación probabilística (Lee et al., 2007). Sin embargo, la experiencia fundamentada en los accidentes industriales indica que los casos más severos han ocurrido en condiciones de vientos de baja intensidad ya que una intensidad alta facilita la dispersión del gas (Crowl y Louvar, 2002; Lee et al., 2007; Patra, 2006). Además, la construcción de edificios cercanos a la fuente de emisión introduce obstrucciones que perturban al fenómeno de dispersión (Duijm et al., 1996). 
En este trabajo se desarrolla un análisis para determinar cual sería el peor escenario con respecto a los factores que afectan la dispersión. El criterio a seguir consiste en asumir que el riesgo depende exclusivamente de los niveles de concentración del gas toxico en los sitios habitados. El riesgo ocurre normalmente en valores acotados de concentración que han sido compilados por organismos internacionales dedicados al control de la seguridad. Así, los análisis realizados sustentan que el peor escenario sería aquel donde la concentración calculada a una cierta distancia de la fuente de emisión sea considerada letal. En las siguientes secciones se plantean los conceptos de los factores de dispersión a analizar, se proponen los modelos matemáticos de dispersión y se analizan los resultados obtenidos.

\section{ANÁLISIS Y MODELADO DE DISPERSIÓN}

Los fenómenos de dispersión de gases en el aire dependen de las condiciones del escenario atmosférico en el que se desarrollan. Las características del escenario de dispersión son determinadas por los factores de dispersión que incluyen la velocidad del viento y la condición atmosférica. El viento es generado por gradientes en la presión y temperatura de la atmósfera haciendo de esta una variable altamente estocástica, cuyos efectos son reconocidos en la existencia de turbulencias. La condición atmosférica representa la capacidad de mezclado entre los gases en dispersión-aire y depende del terreno, la temperatura, la radiación solar, la humedad y la altura. La determinación de las condiciones atmosféricas como variables en los modelos de dispersión es compleja; sin embargo, las condiciones históricas han sido ajustadas y usadas en algunos modelos (Mannan, 2005d). Actualmente las expresiones matemáticas de las condiciones atmosféricas están clasificadas por categorías respecto al efecto que muestran en la dispersión. La forma del terreno influye también en los fenómenos de difusividad incrementándola cuando no represente resistencia al flujo del gas. Así, las condiciones atmosféricas incluyen estos factores y su clasificación ha sido típicamente indicada por Pasquill en 1961 como sigue (Mannan, 2005f): condición atmosférica inestable (A), condición atmosférica semi-inestable $(B)$, condición atmosférica neutra $(D)$, condición atmosférica semi-estable $(E)$, y condición atmosférica estable $(F)$. La siguiente sección discute los modelos de dispersión utilizados.

En el caso de una dispersión pasiva los modelos son planteados con una distribución gaussiana. Los autores de este artículo han realizado previamente un estudio comparativo entre los modelos de Sutton y Pasquill-Gifford encontrando que este último sobreestima la concentración, produciendo una estimación más segura. En el modelo de Pasquill-Gifford se plantea para el caso de una emisión continua con un punto de emisión elevado sobre el nivel del suelo, dado por(Mannan, 2005c):

$C_{\langle x, y, z\rangle}=\frac{Q}{2 \pi v \sigma_{z} \sigma_{y}} \exp \left(-\frac{y^{2}}{2 \sigma_{y}{ }^{2}}\right)\left[\exp \left(-\frac{(z-H)^{2}}{2 \sigma_{z}{ }^{2}}\right)+\exp \left(-\frac{(z+H)^{2}}{2 \sigma_{z}{ }^{2}}\right)\right]$

donde $C_{\langle x, y, z\rangle}$ es la concentración de la dispersión en las coordenadas $x, y$ y $z, Q$ es el flujo másico del gas, $H$ es la altura de la fuente de emisión, $v$ es la velocidad del viento, $y$ y $z$ son coordenadas de los puntos de análisis de la concentración, y $\sigma_{y}$ y $\sigma_{z}$ son parámetros de dispersión definidos por Gifford que son específicos para el tipo de terreno y condición atmosférica (Mannan, 2005d).

En el caso de dispersión de gases densos, los modelos analíticos tienden a ser extremadamente complejos ya que deben incluir, por ejemplo, los efectos de temperatura y humedad. Sin embargo, existen modelos simples tales como Germeles y DENZ que han mostrado ser eficientes y representativos en situaciones prácticas (Ermak et al., 1982). De estos modelos se selecciona el de Germeles debido a que sobreestima la concentración de la dispersión en análisis numéricos realizados por los autores. La concentración por el modelo de Germeles es calculada como:

$C_{\langle x, y, z\rangle}=\frac{Q}{v \sigma_{z} L \sqrt{2 \pi}} \exp \left(-\frac{1}{2}\left(\frac{z}{\sigma_{z}}\right)^{2}\right)\left[\operatorname{erf}\left(-\frac{L-2 y}{\sigma_{y} 2 \sqrt{2}}\right)+\operatorname{erf}\left(-\frac{L+2 y}{\sigma_{y} 2 \sqrt{2}}\right)\right]$ 
donde $L$ es el ancho de la fuente de emisión. A continuación se describe el uso de los modelos de dispersión incluyendo la selección de valores numéricos utilizados. Cabe agregar que los modelos gaussianos simplifican el modelo matemático, evitando el modelo complejo de la dispersión cuya dificultad, tanto del modelado como de la solución, se magnifica en condiciones urbanas. Los cálculos relacionados a dispersión que demanden una mejor precisión o un análisis a microescala podrían usar los modelos CFD (Blocken et al., 2007; Gilham et al., 2000).

Una limitante importante en los modelos matemáticos es la existencia de indeterminaciones cuando las velocidades del viento tienden a cero. De hecho, los autores indican que el cálculo de concentraciones a bajas velocidades presenta predicciones cuestionables. Por esta razón, la velocidad del viento mínima sugerida es un valor mayor y cercano a $1 \mathrm{~m} \cdot \mathrm{s}^{-1}$. Estadísticamente, la velocidad del viento máxima detectada en accidentes es cercana a $6 \mathrm{~m} \cdot \mathrm{s}^{-1}$ (Lee et al., 2007; Wiekema, 1984). Por otro lado, las condiciones atmosféricas analizadas son la inestable $(A)$ y estable $(F)$ por representar características extremas opuestas entre sí mientras que la topografía del terreno ha sido considerada en sus formas urbana y rural. La Tabla 1 indica los parámetros de dispersión para las condiciones atmosféricas en distintos terrenos a una distancia $x$.

Para realizar el estudio comparativo de la dispersión pasiva se usa el escenario mostrado en (Crowl y Louvar, 2002). La emisión ocurre en una chimenea que emite $\mathrm{SO}_{2}$ con flujo másico de $0.08 \mathrm{~kg} \cdot \mathrm{s}^{-1}$. La altura del punto de emisión en la chimenea es $60 \mathrm{~m}$ sobre el nivel del suelo y el punto de análisis $z$ se encuentra a $55 \mathrm{~m}$ de altura. Para el caso de dispersión de gases densos, se plantea la emisión de cloro como se indica en (CCPS, 1989). El derrame en fase líquida del compuesto tóxico genera un radio de $2.74 \mathrm{~m}$. La dispersión del gas tóxico es de un flujo másico constante de $3.075 \mathrm{~kg} \cdot \mathrm{s}^{-1} \mathrm{y}$ el análisis de la concentración es realizado al nivel del suelo. Para ambos casos de dispersión se analizan los resultados de la concentración únicamente en las coordenadas $x$ y $z$ por motivos de simplicidad en la representación. A continuación se muestra un análisis de los resultados obtenidos para ambos tipos de dispersión.

\section{RESULTADOS Y DISCUSIÓN}

Al realizar las estimaciones de concentración con dispersión pasiva en un terreno urbano se observó que las velocidades del viento bajas generan concentraciones mayores. El comportamiento para todas las velocidades de la dispersión pasiva indica la existencia de un máximo seguido de un decaimiento pronunciado en la concentración. En el terreno urbano, la condición atmosférica inestable señala que la distancia de la concentración máxima al punto de emisión es menor que en la condición atmosférica estable. Lo anterior indica que la dispersión es lenta en la condición atmosférica estable. Además, el decaimiento es más pronunciado en condición atmosférica inestable lo cual se justifica en la realidad por la existencia de una mayor turbulencia. La Figura 1 muestra los resultados para la dispersión en terreno urbano con velocidades de $1.5 \mathrm{~m} \cdot \mathrm{s}^{-1}$ y $6 \mathrm{~m} \cdot \mathrm{s}^{-1}$ que fueron usadas en este estudio tanto para condición atmosférica inestable, Figura 1a, como para condición atmosférica inestable, Figura $1 \mathrm{~b}$.

Tabla 1: Ecuaciones para condiciones atmosféricas $(A)$ y $(F)$ en distintas topografías (Crowl y Louvar, 2002).

\begin{tabular}{|l|l|l|l|}
\cline { 3 - 4 } \multicolumn{2}{c|}{} & Terreno urbano & Terreno rural \\
\hline Condición Atmosférica Estable $(\mathrm{F})$ & $\sigma_{y}$ & $0.11 x(1+0.0004 x)^{-0.5}$ & $0.04 x(1+0.0001 x)^{-0.5}$ \\
\cline { 2 - 4 } & $\sigma_{z}$ & $0.08 x(1+0.0015 x)^{-0.5}$ & $0.016 x(1+0.0003 x)^{-1}$ \\
\hline \multirow{2}{*}{ Condición Atmosférica Inestable $(\mathrm{A})$} & $\sigma_{y}$ & $0.32 x(1+0.0004 x)^{-0.5}$ & $0.22 x(1+0.0001 x)^{-0.5}$ \\
\cline { 2 - 4 } & $\sigma_{z}$ & $0.24 x(1+0.0001 x)^{+0.5}$ & $0.20 x$ \\
\hline
\end{tabular}

Los resultados obtenidos al calcular concentraciones de dispersión pasiva en terreno rural mostraron, de igual forma, que las velocidades del viento bajas generan concentraciones mayores. Además, la distancia de la concentración máxima al punto de emisión es mayor que la presentada por el terreno urbano debido a que no existen obstrucciones a la dispersión. Asimismo, las turbulencias de la condición atmosférica inestable describen que la distancia del punto de emisión al punto de concentración máxima es menor a la obtenida en la condición atmosférica estable. La condición at- 
mosférica inestable en terreno rural muestra un decaimiento pronunciado en la concentración. Sin embargo, la condición atmosférica estable genera un decremento menor en la concentración conforme se aleja de la fuente de emisión, indicando con esto una dispersión lenta. La Figura 2 describe la dispersión en terreno rural en las condiciones atmosféricas inestable y estable.

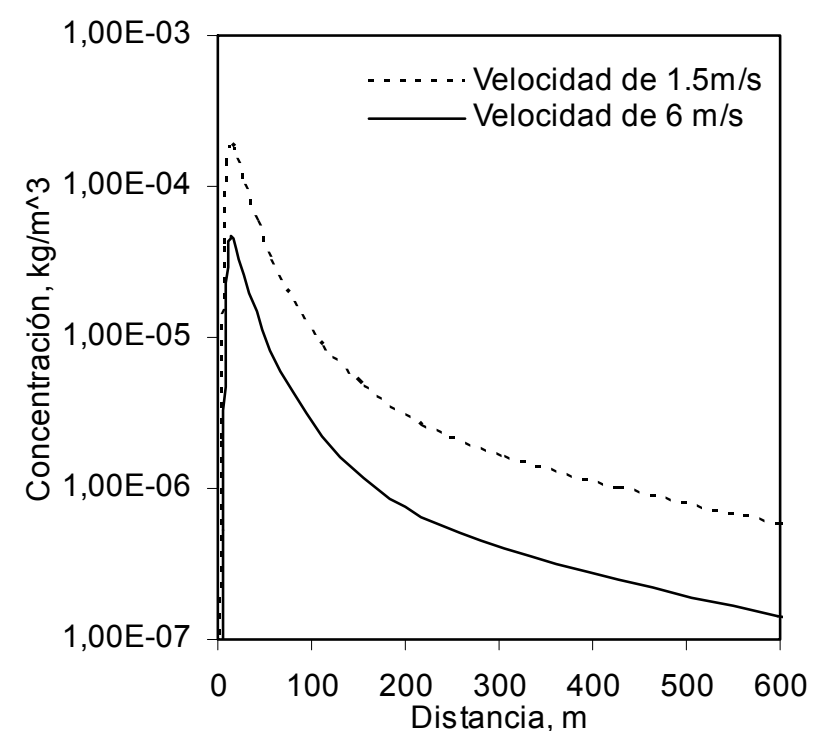

(a)

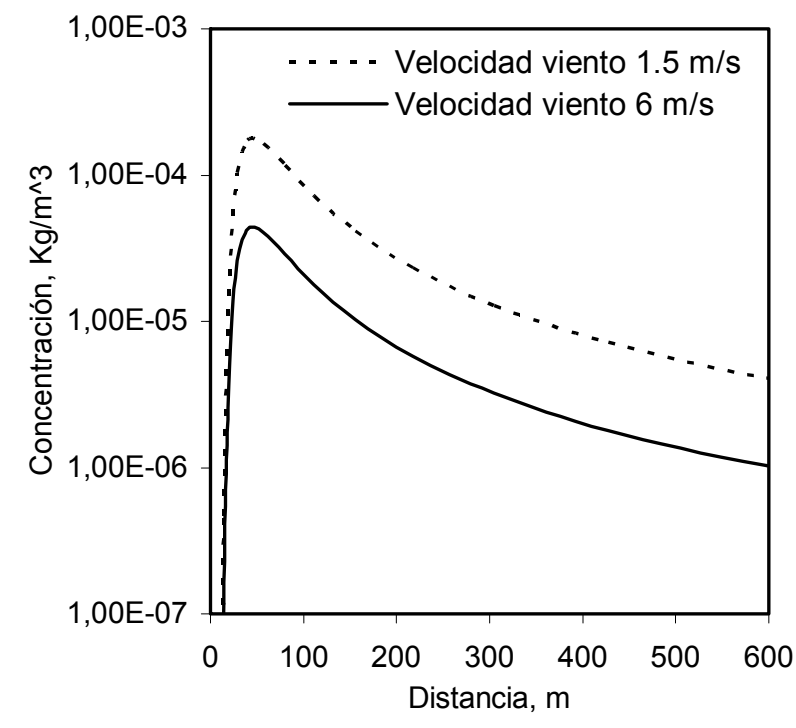

(b)

Fig. 1: Dispersión pasiva en terreno urbano con condición atmosférica inestable (a) y estable (b).

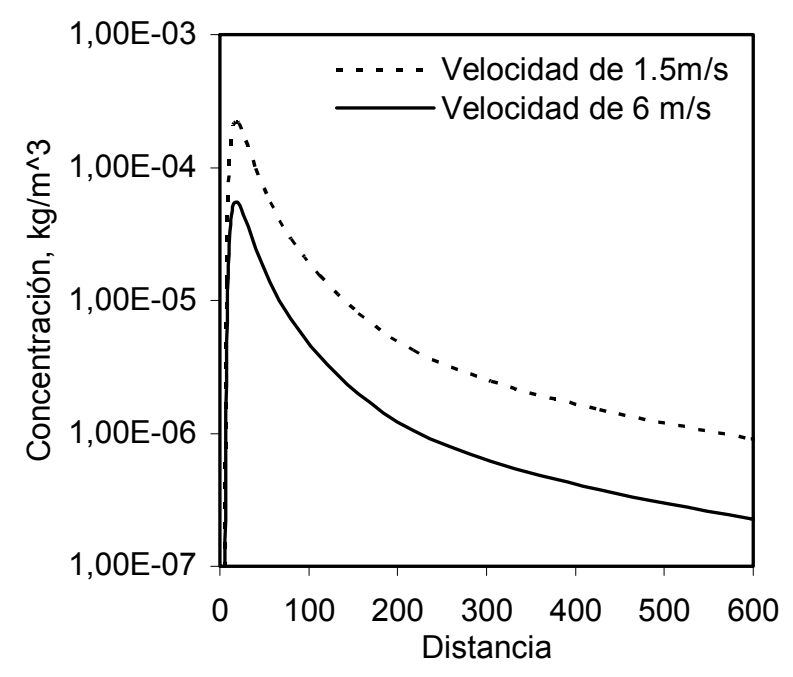

(a)

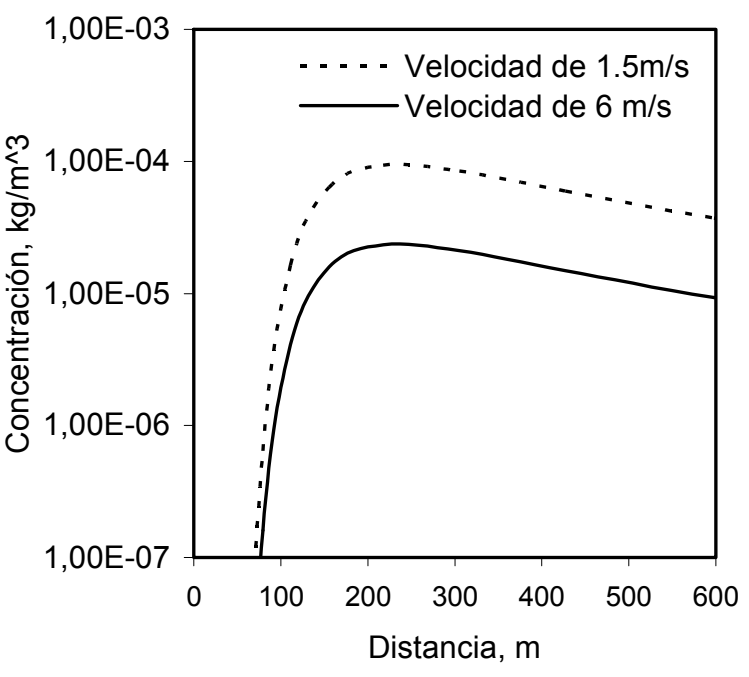

(b)

Fig. 2: Dispersión pasiva en terreno rural con condición atmosférica inestable (a) y estable (b).

El caso de los gases densos, y particularmente en este caso de estudio, no genera una concentración máxima en su dispersión. La dispersión de gases densos es descrita por un decaimiento total de la concentración hasta el punto que inicia la curvatura en la dispersión, comenzando así una dispersión pasiva. La dispersión de gases densos en terreno urbano muestra concentraciones mayores a velocidades del viento bajas y el inicio de la dispersión pasiva cercano a la fuente de emisión. Además, la condición atmosférica inestable describe valores menores de concentración con respecto a la condición atmosférica estable. Lo anterior explica que, al ser mayor la turbulencias en la condición atmosférica inestable, la dispersión es más rápida que en la condición atmosférica estable. El comportamiento de la dispersión de gases densos en terreno urbano con condiciones atmosféricas inestable y estable se muestra en la Figura 3. 
La dispersión de gases densos en terreno rural describe valores de concentraciones mayores a velocidades bajas. Además, por no existir obstrucciones en la dispersión en terreno rural la distancia del punto de emisión al inicio de la curvatura en la dispersión es mayor que en el terreno urbano. Los valores de la concentración son inclusive mayores que los valores de la condición atmosférica inestable al ser la turbulencia mucho menor en la condición atmosférica estable. La Figura 4 muestra la dispersión de gases densos en un terreno rural a condición atmosférica inestable y estable.

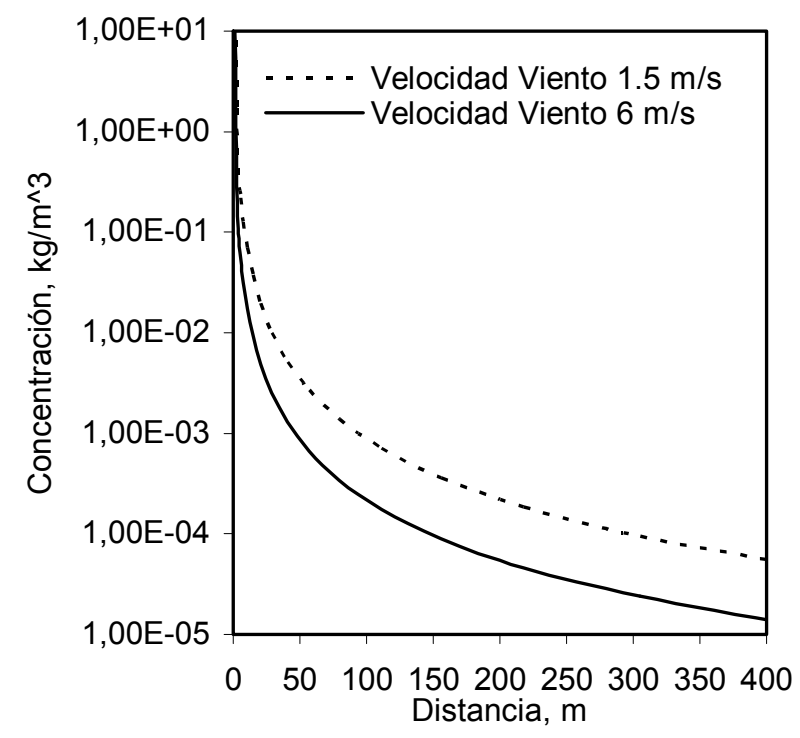

(a)

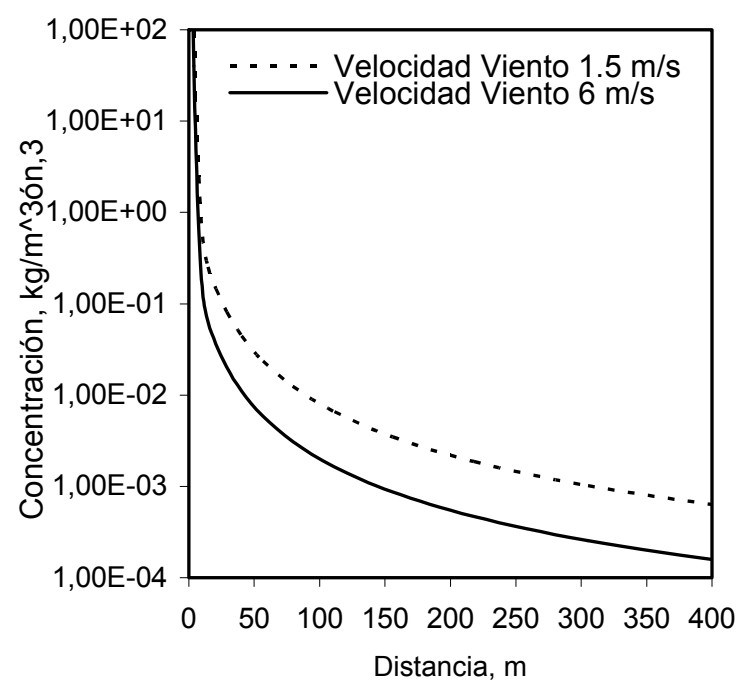

(b)

Fig. 3: Dispersión densa en terreno urbano con condición atmosférica inestable (a) y estable (b).

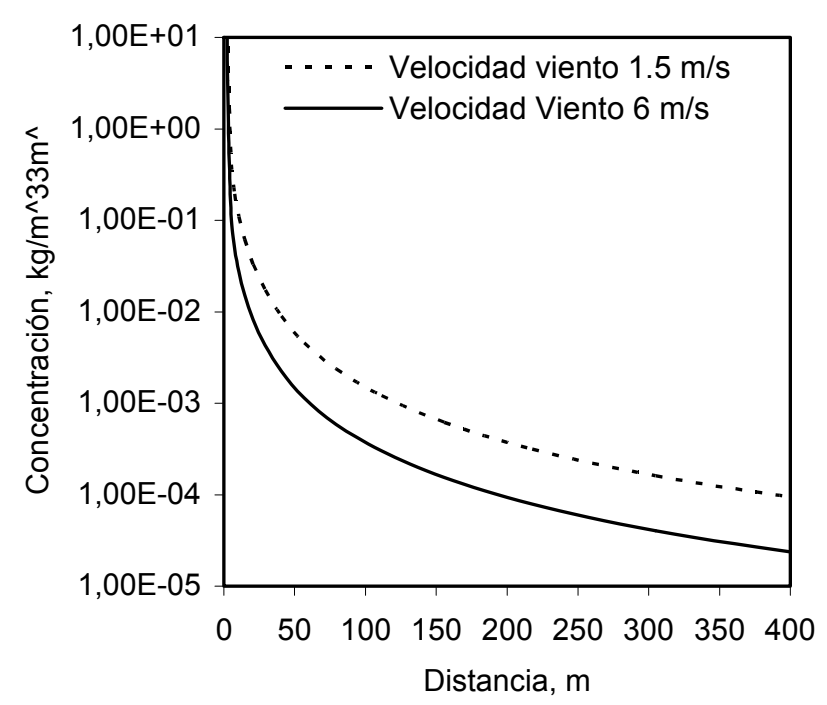

(a)

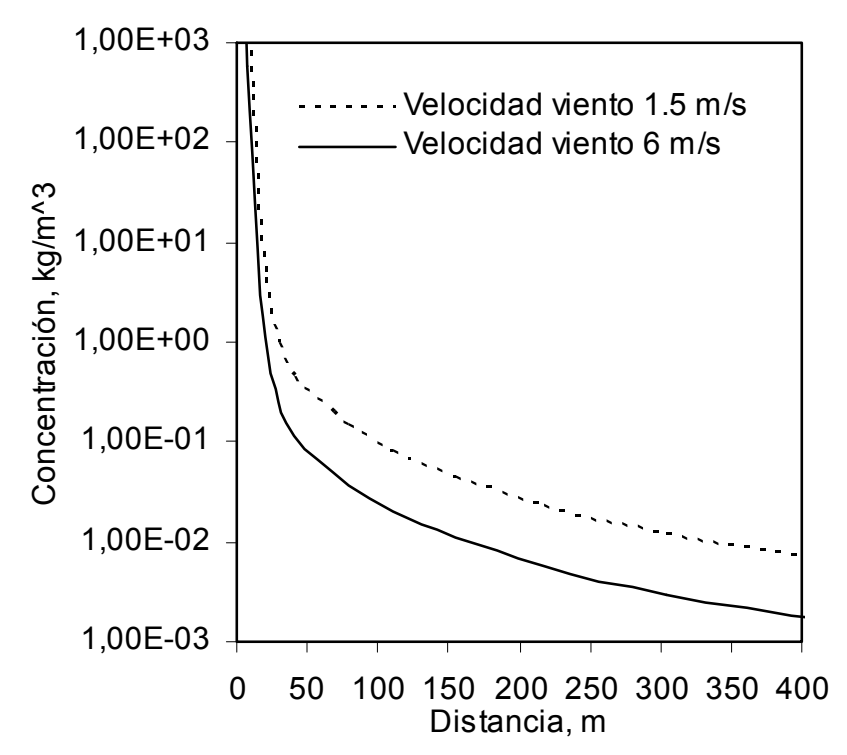

(b)

Fig. 4: Dispersión densa en terreno rural con condición atmosférica inestable (a) y estable (b).

Las dispersiones pasiva y densa ratifican la existencia de altas concentraciones a una velocidad del viento baja o en "calma", entre 1 y $2 \mathrm{~m} \cdot \mathrm{s}^{-1}$ (Wiekema, 1984), debido a la poca transferencia de momentum entre la dispersión. La condición atmosférica inestable manifiesta una rápida difusión al inicio de la dispersión debido a las turbulencias existentes. Asimismo, el terreno urbano describe, en la gráfica de dispersión, la obstrucción a la difusión del gas. En los casos donde el punto de análisis se encuentra debajo o sobre el punto de emisión, existe un máximo en la concentración indicando inmersión en la dispersión. La tendencia mostrada anteriormente fue ratificada con otros casos de 
estudio desarrollado por los autores en donde se consideraron diferentes flujos, alturas de emisión y tamaños de la fuente de emisión para validar las conclusiones de la siguiente sección.

\section{CONCLUSIONES}

El análisis presentado muestra que los factores de dispersión tienen una influencia similar para los dos tipos de dispersiones de gases, i.e. pasiva y densa. Los resultados indican la existencia de concentraciones más altas cuando la velocidad del viento es en condición de calma. Por otro lado, la condición atmosférica estable en terreno rural describe una concentración máxima de la dispersión a una distancia alejada del punto de emisión. Así, la sugerencia es que el peor escenario requerido en análisis de riesgo debe considerar una velocidad baja y una condición atmosférica estable en terreno rural. Además, los modelos de Pasquill-Gifford y Germeles predicen valores de concentración mayores a los obtenidos en otros modelos resultando ideales para la obtención de diseños más seguros como por ejemplo en la distribución óptima de las unidades de proceso y facilidades industriales.

\section{AGRADECIMIENTOS}

Los autores agradecen el apoyo de la Universidad de Texas A\&M, CONACYT y DGEST.

\section{REFERENCIAS}

Blocken, B., J. Carmeliet y T. Stathopoulos; CFD evaluation of wind speed conditions in passages between parallel buildings-effect of wall-function roughness modifications for the atmospheric boundary layer flow, Journal of Wind Engineering and Industrial Aerodynamics: In Press (2007).

CCPS; Case Studies, In Guidelines for chemical process quantitative risk analysis, Center for Chemical Process Safety, AIChE, pp. 580-590 (1989).

Crowl, D.A. y J.F. Louvar; Toxic Release and Dispersion Models, In Chemical process safety, fundamentals with applications, $2^{\mathrm{a}}$ edición, Prentice Hall International Series in the Physical and Chemical Engineering Sciences, Cap. 5, pp. 180 -210, New Jersey, EE.UU. (2002).

Dandrieux-Bony, A., J.-P. Dimbour y G. Dusserre; A simple model for calculating chlorine concentrations behind a water spray in case of small releases, Journal of Loss Prevention in the Process Industries: 18 (4-6), 245-253 (2005).

Duijm, N. J., S. Ott y M. Nielsen; An evaluation of validation procedures and test parameters for dense gas dispersion models, Journal of Loss Prevention in the Process Industries: 9 (5), 323-338 (1996).

Ermak, D.L., S.T. Chan, D.L. Morgan y L.K. Morris; A comparison of dense gas dispersion model simulations with burro series LNG spill test results, Journal of Hazardous Materials: 6 (1-2), 129-160 (1982).

Gilham, S., D. M. Deaves y P. Woodburn; Mitigation of dense gas releases within buildings: validation of CFD modelling, Journal of Hazardous Materials: 71 (1-3), 193-218 (2000).

Jonsson, L., E. Karlsson y L. Thaning; Toxic gas clouds: Effects and implications of dry deposition on concentration, Journal of Hazardous Materials: 124 (1-3), 1-18 (2005).

Lee, J.-H., R. Vázquez-Román, S. Jung y S. Mannan; An approach to incorporate Long-term meteorological data in dispersion analysis to estimate directional risk, Chemical Engineering Journal: Manuscrito enviado, por confirmar aceptación (2007).

Mannan, S.; Dense Gas Dispersion, In Lee's Lost Prevention in the Process Industries, 3ra edición, Elsevier Butterworth-Heinemann Publications, Vol. 1, Cap. 15, pp. 155-160 (2005a). 
Mannan, S.; Passive Dispersion, In Lee's Lost Prevention in the Process Industries, 3ra edición, Elsevier Butterworth-Heinemann Publications, Vol. 1, Cap. 15, pp. 102-103 (2005b).

Mannan, S.; Passive Dispersion: Models, In Lee's Lost Prevention in the Process Industries, 3ra edición, Elsevier Butterworth-Heinemann Publications, Vol. 1, Cap. 15, pp. 103-116 (2005c).

Mannan, S.; Passive Dispersion: Dispersion Parameters, In Lee's Lost Prevention in the Process Industries, 3ra edición, Elsevier Butterworth-Heinemann Publications, Vol. 1, Cap. 15, pp. 121-134, (2005d).

Mannan, S.; Lee's Lost Prevention in the Process Industries, 3ra edición, Vol. 1, Cap. 15, 167-191. Elsevier Butterworth-Heinemann Publications, (2005e).

Mannan, S.; Stability Clasification, In Lee's Lost Prevention in the Process Industries, 3ra edición, Elsevier Butterworth-Heinemann Publications, Vol. 1, Cap. 15, pp. 87-92, (2005f).

Park, O.-H. y M.-G. Seok; Selection of an appropriate model to predict plume dispersion in coastal areas, Atmospheric Environment: 41 (29), 6095-6101 (2007).

Patra, A. K.; Influence of wind speed profile and roughness parameters on the downwind extension of vulnerable zones during dispersion of toxic dense gases, Journal of Loss Prevention in the Process Industries: 19 (5), 478-480 (2006).

Sharan, M. y S.G. Gopalakrishnan; Bhopal gas accident: a numerical simulation of the gas dispersion event, Enviromental Modelling and Software: 12, 135-141 (1997).

Sklavounos, S. y F. Rigas; Validation of turbulence models in heavy gas dispersion over obstacles, Journal of Hazardous Materials: 108 (1-2), 9-20 (2004).

Thomson, L. C. y otros cinco autores; An improved algorithm for locating a gas source using inverse methods, Atmospheric Environment: 41 (6), 1128-1134 (2007).

Turner, D.B.; A difusion model for an urban area, Journal of Applied Meteorology: 3 (February), 83-91 (1964).

Vázquez-Román, R., J.-H. Lee, S. Jung y S. Mannan; Optimal facility Layout under toxic release in existing plants, Computers \& Chemical Engineering: Manuscrito enviado, por confirmar aceptación, (2007).

Wiekema, B. .; Vapour cloud explosions- An analysis based on accidents Part II, Journal of Hazardous Materials: 8, 313-329 (1984).

Yegnan, A., D.G. Williamson y A.J. Graettinger; Uncertainty analysis in air dispersion modeling, Environmental Modelling \& Software: 17 (7), 639-649 (2002). 